\title{
Logarithmic Singularity of Specific Heat in Liquid Helium II at the $\lambda$ Point
}

\author{
Shosuke Sasaki
}

Published online: 5 June 2007

(C) Springer Science+Business Media, LLC 2007

\begin{abstract}
The experimental width of the second sound peak in Brillouin scattering is less than the instrumental width. It is also less than the width of the first sound peak near the $\lambda$ point. The theoretical width of the second sound peak becomes infinitely large at the $\lambda$ point in the traditional viewpoint. This discrepancy suggests that the second sound peak detected in Brillouin scattering is an elementary excitation. We calculate the specific heat of liquid helium near the $\lambda$ point by considering the second sound peak. The calculated temperature-dependence of the specific heat has a logarithmic divergence at the $\lambda$ point. Furthermore, the calculated values of the specific heat agree well with data derived from experiments.
\end{abstract}

PACS $67.40 . \mathrm{Kh} \cdot 67.40 . \mathrm{Db} \cdot 67.40 .-\mathrm{w} \cdot 64.60 . \mathrm{Fr}$

\section{Introduction}

The specific heat of liquid helium was calculated using the Landau theory [1, 2]; the results agree well with experimental data near zero temperature. However, the calculated values differ greatly from the experimental data obtained near the $\lambda$ point. Bendt et al. [1] and Brooks and Donnelly [2] calculated the specific heat and other thermodynamic functions of superfluid helium on the basis of the Landau theory. Their result for specific heat has no logarithmic divergence at the $\lambda$ point. On the other hand, experimental data [3,4] show the logarithmic divergence. Therefore, we need to study a new theory of specific heat. We focus on the low-energy excitation "second sound peak" appearing in the Brillouin scattering [5-8].

The second sound mode is considered to be a thermal wave mode and its theoretical width becomes infinitely large at the $\lambda$ point in the traditional viewpoint.

S. Sasaki $(\bowtie)$

Shizuoka Institute of Science and Technology, 2200-2 Toyosawa, 437-8555, Fukuroi, Japan

e-mail: sasaki@ns.sist.ac.jp 
However, its experimental width is very narrow in spectra of Brillouin scatterings. A study of [7] reveals that the experimental width of the second sound peak is nearly equal to $1.5 \mathrm{MHz}$, which is constant for variation of the temperature value. This width is nearly equal to the instrumental width. Therefore, the intrinsic width is less than 1.5 MHz. This temperature dependence of the width does not agree with the result obtained using the Landau theory. The experimental result of Vaughan, Vinen and Palin [8] shows that the width of the second sound peak is smaller than about 1/10 of the first sound width at the temperature $T=T_{\lambda}-0.5 \mathrm{mK}$. Therefore, the lifetime of the second sound mode is longer by about 10 times than that of the first sound mode. For that reason, difficulties exist in the traditional interpretation that the second sound mode detected in Brillouin scattering is a multi-excitation mode of the first sound.

In this paper, we reinterpret this second sound peak of Brillouin scattering as an excitation from Bose-Einstein condensate in superfluid helium, and calculate the specific heat of liquid helium considering the this second sound peak. Then, we obtain the logarithmic term in the calculation result.

\section{Excitation Energy Near the $\lambda$ Point}

The superfluid component in a dilute atomic gas is well known to be a Bose-Einstein condensate. For liquid helium, the superfluid component is a background flow in Landau theory. Hence, we reexamine the nature of the superfluid component of liquid helium. The total Hamiltonian $H$ of liquid helium is $H=\sum_{\mathbf{p}} \frac{\mathbf{p}^{2}}{2 m} a_{\mathbf{p}}^{*} a_{\mathbf{p}}+$ $\frac{1}{2 V} \sum_{\mathbf{p}, \mathbf{q}, \mathbf{k}} g(\mathbf{k}) a_{\mathbf{p}+\mathbf{k}}^{*} a_{\mathbf{q}-\mathbf{k}}^{*} a_{\mathbf{p}} a_{\mathbf{q}}$ where $m$ is the mass of a helium atom, $a_{\mathbf{p}}^{*}$ and $a_{\mathbf{p}}$ are the creation and annihilation operators, and $g(\mathbf{k})$ is the interatomic potential. An operator $U$ is defined as the unitary transformation that diagonalizes the total Hamiltonian $H$. Although the explicit form of $U$ is unknown, we can discuss many properties of $U$, for example, number conservation, Galilean invariance, mass conservation, and momentum conservation. Consequently, all the eigenstates of $H$ are expressed using the transformation from free states of helium atoms to |eigenstate $\rangle=$ $U a_{\mathbf{p}_{1}}^{*} a_{\mathbf{p}_{2}}^{*} a_{\mathbf{p}_{3}}^{*} \ldots a_{\mathbf{p}_{N}}^{*}|0\rangle$. A new creation operator is defined as $A_{\mathbf{p}}^{*}=U a_{\mathbf{p}}^{*} U^{-1}$, which indicates a "dressed boson operator" with an interaction cloud. The eigenstate is expressed as

$$
\mid \text { eigenstate }\rangle=A_{\mathbf{p}_{1}}^{*} A_{\mathbf{p}_{2}}^{*} A_{\mathbf{p}_{3}}^{*} \ldots A_{\mathbf{p}_{N}}^{*} U|0\rangle=A_{\mathbf{p}_{1}}^{*} A_{\mathbf{p}_{2}}^{*} A_{\mathbf{p}_{3}}^{*} \ldots A_{\mathbf{p}_{N}}^{*}|0\rangle .
$$

Therein, we have used the equation $U|0\rangle=|0\rangle$ because the vacuum state $|0\rangle$ is an eigenstate of $H$. Consequently, all the eigenstates of liquid helium are expressed as direct products of the dressed boson operators. The eigenenergy $E$ is therefore expressible with the number distribution $n_{\mathbf{p}}=A_{\mathbf{p}}^{*} A_{\mathbf{p}}$ of the dressed bosons in momentum space: $E=E\left(\left\{n_{\mathbf{p}}\right\}\right)$.

The total Hamiltonian $H$ is Galilean covariant; therefore, the diagonal form of $H$ is the sum of the kinetic energy $K$ of the center of mass and Galilean invariant terms $X$, as $H=K+X$ where $K=\frac{1}{2 M} \sum_{\mathbf{p}} \mathbf{p} n_{\mathbf{p}} \cdot \sum_{\mathbf{q}} \mathbf{q} n_{\mathbf{q}}=-\frac{1}{2 M} \sum_{\mathbf{p}, \mathbf{q}} \frac{1}{2}(\mathbf{p}-\mathbf{q})^{2} n_{\mathbf{p}} n_{\mathbf{q}}+$ $\sum_{\mathbf{p}} \frac{\mathbf{p}^{2}}{2 m} n_{\mathbf{p}}$. In that derivation, we used $N=\sum_{\mathbf{q}} a_{\mathbf{q}}^{*} a_{\mathbf{q}}=\sum_{\mathbf{q}} A_{\mathbf{q}}^{*} A_{\mathbf{q}}=\sum_{\mathbf{q}} n_{\mathbf{q}}$ because of total number conservation. In addition, $M$ is the total mass of liquid helium, 
and $N$ and $m=M / N$ respectively denote the total number and the mass of helium atom. Thereby, the diagonal form of the total Hamiltonian $H$ is expressed as $H=K+X=\sum_{\mathbf{p}} \frac{\mathbf{p}^{2}}{2 m} n_{\mathbf{p}}-\frac{1}{2 M} \sum_{\mathbf{p}, \mathbf{q}} \frac{1}{2}(\mathbf{p}-\mathbf{q})^{2} n_{\mathbf{p}} n_{\mathbf{q}}+X=\sum_{\mathbf{p}} \frac{\mathbf{p}^{2}}{2 m} n_{\mathbf{p}}+($ Galilean invariant terms). Therein Galilean invariant terms are described only by the relative momenta of dressed bosons. Hence, we obtain the total energy using an arbitrary function $f$ as the following:

$$
E=\sum_{\mathbf{p}} \frac{\mathbf{p}^{2}}{2 m} n_{\mathbf{p}}+\frac{1}{N} \sum_{\mathbf{p}, \mathbf{q}} f(\mathbf{p}-\mathbf{q}) n_{\mathbf{p}} n_{\mathbf{q}}
$$

The ground state energy is $E_{G}=f(0) N$ when all the dressed bosons have momentum of zero $\left(n_{0}=N\right)$. Therefore, $-f(0)$ is the latent heat per atom at zero Kelvin. The first excited energy is obtained for the number distribution $\left\{n_{0}=N-1, n_{\mathbf{p}}=1\right\}$ :

$$
\begin{aligned}
E & =\frac{\mathbf{p}^{2}}{2 m}+\frac{1}{N}\left[f(0)(N-1)^{2}+f(\mathbf{p})(N-1)+f(-\mathbf{p})(N-1)+f(\mathbf{p}-\mathbf{p})\right] \\
& \approx f(0) N+\frac{\mathbf{p}^{2}}{2 m}+2(f(\mathbf{p})-f(0))
\end{aligned}
$$

We have used $1 / N \approx 0$ and the spherical symmetric property of the function $f(\mathbf{p})$. If we take the functional form as $f(\mathbf{p})=\frac{1}{2}\left(\epsilon_{\mathbf{p}}^{0}-\mathbf{p}^{2} /(2 m)\right)+f(0)$ where $\epsilon_{\mathbf{p}}^{0}$ is the elementary excitation energy at zero Kelvin, then we obtain the first excited energy of the total system as $E=E_{G}+\epsilon_{\mathbf{p}}^{0}$. This excitation energy $E-E_{G}$ is in agreement with the Landau theory. It should be noted here that the functional form of $f(\mathbf{p})$ has been determined from experimental data of the elementary excitation energy $\epsilon_{\mathbf{p}}^{0}$ and the latent heat at the temperature $T=0$. Therefore, the present theory is equivalent to the Landau theory near zero Kelvin. The energy of one dressed boson with momentum $\mathbf{p}$ is the energy increase when a single dressed boson is added to the system:

$$
\omega_{\mathbf{p}}=\frac{\delta E}{\delta n_{\mathbf{p}}}=\frac{\mathbf{p}^{2}}{2 m}+\frac{2}{N} \sum_{\mathbf{q}} f(\mathbf{p}-\mathbf{q}) n_{\mathbf{q}}-\frac{1}{N^{2}} \sum_{\mathbf{s}, \mathbf{t}} f(\mathbf{s}-\mathbf{t}) n_{\mathbf{s}} n_{\mathbf{t}} .
$$

The number distribution of dressed bosons is given as $n_{\mathbf{p}}=1 /\left[\exp \left(\left(\omega_{\mathbf{p}}-\mu\right) /\right.\right.$ $\left.\left.\left(k_{B} T\right)\right)-1\right]$, where $\mu$ is the chemical potential, and $k_{B}$ is the Boltzmann constant. The chemical potential $\mu$ is nearly equal to $\omega_{0}$ for $T<T_{\lambda}$. It follows that $n_{0}$ is a macroscopic number for $T<T_{\lambda}$. Consequently, Bose-Einstein condensation of the dressed bosons appears. The energy of excitation from the Bose condensate is $\epsilon_{\mathbf{p}}=\omega_{\mathbf{p}}-\omega_{0}=\frac{\mathbf{p}^{2}}{2 m}+\frac{2}{N} \sum_{\mathbf{q}}(f(\mathbf{p}-\mathbf{q})-f(-\mathbf{q})) n_{\mathbf{q}}$ which depends upon the temperature value because the number distribution function $n_{\mathbf{q}}$ depends on $T$. For small momenta $p<q_{A}$ and $q<q_{A}$, we can use an approximate form for $f: f(\mathbf{p}-\mathbf{q}) \approx$ $\frac{1}{2} c|\mathbf{p}-\mathbf{q}|+f(0)+\operatorname{Order}(\mathbf{p}-\mathbf{q})^{2}$ where $c$ is the phonon velocity at zero Kelvin. This approximation is derived from the equation $f(\mathbf{p})=\frac{1}{2}\left(\epsilon_{\mathbf{p}}^{0}-\mathbf{p}^{2} /(2 m)\right)+f(0)$ using $\epsilon_{\mathbf{p}}^{0}=c|\mathbf{p}|$ for a small momentum. Substitution of this approximation into (3) gives the energy of the dressed boson as the following expression:

$$
\omega_{\mathbf{p}}=\frac{\mathbf{p}^{2}}{2 m}+\frac{n_{0}}{N} c|\mathbf{p}|+\frac{V}{N(2 \pi \hbar)^{3}} \int_{q=0}^{q_{A}} \iint c|\mathbf{p}-\mathbf{q}| n_{\mathbf{q}} \mathrm{d} \cos \theta \mathrm{d} \phi q^{2} \mathrm{~d} q
$$




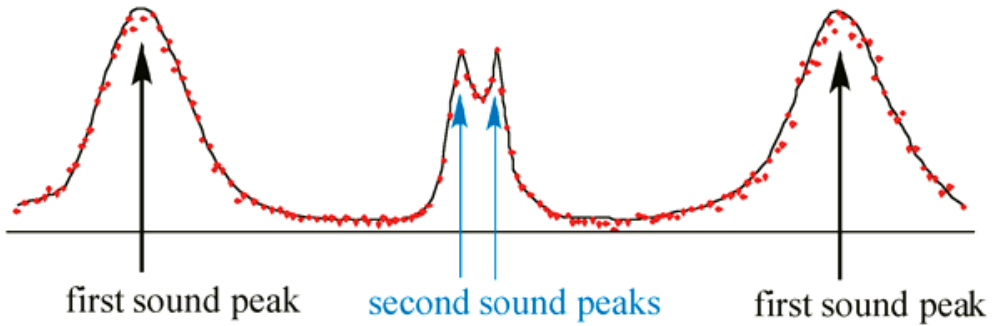

Fig. 1 (Color on-line) Spectrum in Brillouin scattering of laser for liquid helium II

$$
+\frac{2 V}{N(2 \pi \hbar)^{3}} \int_{q=q_{A}}^{\infty} \iint f(\mathbf{p}-\mathbf{q}) n_{\mathbf{q}} \mathrm{d} \cos \theta \mathrm{d} \phi q^{2} \mathrm{~d} q+(\text { constant value })
$$

where

$$
\begin{aligned}
& \int_{0}^{q_{A}} \iint c|\mathbf{p}-\mathbf{q}| n_{\mathbf{q}} \mathrm{d} \cos \theta \mathrm{d} \phi q^{2} \mathrm{~d} q \\
& =2 \pi \int_{0}^{q_{A}} \int_{-1}^{1} c \sqrt{p^{2}-2 p q t+q^{2}} \\
& \quad \times \mathrm{d} t n_{q} q^{2} \mathrm{~d} q=2 \pi \int_{0}^{q_{A}} c \frac{|p+q|^{3}-|p-q|^{3}}{3 p q} n_{q} q^{2} \mathrm{~d} q \\
& =\operatorname{Order}\left(p^{2}\right)+(\text { constant value }), \\
& \int_{q_{A}}^{\infty} \iint f(\mathbf{p}-\mathbf{q}) n_{\mathbf{q}} \mathrm{d} \cos \theta \mathrm{d} \phi q^{2} \mathrm{~d} q \\
& =\int_{q_{A}}^{\infty} \iint\left[f(-\mathbf{q})+\alpha \mathbf{p} \cdot \mathbf{q}+\beta p^{2}+\cdots\right] n_{\mathbf{q}} \mathrm{d} \cos \theta \mathrm{d} \phi q^{2} \mathrm{~d} q \\
& =\operatorname{Order}\left(p^{2}\right)+(\text { constant value }) .
\end{aligned}
$$

Because these integrated values depend on $p^{2}, \omega_{\mathbf{p}}-\omega_{0}$ is

$$
\omega_{\mathbf{p}}-\omega_{0}=\frac{n_{0}}{N} c p+\operatorname{Order}\left(p^{2}\right),
$$

for a small momentum $p$ (see [9]). Therefore, the velocity of a dressed boson becomes $n_{0} c / N$, which approaches zero at the $\lambda$ point. This property of the excitation from the condensate resembles that of the second sound mode in Brillouin scatterings [5-8], because the velocity of the second sound also becomes zero at the $\lambda$ point.

As Fig. 1 shows, the width of the second sound peak is smaller than about $1 / 10$ of the first sound width at the temperature $T=T_{\lambda}-0.5 \mathrm{mK}$ (see [8]). Therefore, the lifetime of the second sound mode is longer by about 10 times than that of the first sound mode. Hence, difficulties appear in the traditional interpretation that the second sound mode detected in Brillouin scattering is a multi-excitation mode of the first sound. Consequently, we adopt a new viewpoint that the second sound mode detected in Brillouin scattering near the $\lambda$ point is the excitation from the condensate. This viewpoint facilitates reformulation of the thermodynamics of liquid helium. 


\section{Specific Heat}

We apply our viewpoint mentioned above, and recalculate the specific heat. According to BD theory [2], integrations are separable into four momentum regions: phonon, maxon, roton, and higher momentum. We also use four functional forms, two of which are identical functions for the roton region and high-momentum region to those in the BD theory. For the phonon region, we use the second sound mode, the functional form of which is discussed below. The function parameters in the maxon region are determined such that the excitation energy and its tangent are connected continuously to both neighbor curves, as in BD theory. Experimental results described in reference [10] indicate that the second sound velocity near the $\lambda$ point depends on the temperature as $c_{2}\left(1-\left(T / T_{\lambda}\right)\right)^{1 / 3}+D\left(1-\left(T / T_{\lambda}\right)\right)^{1 / 2}$. According to our viewpoint for the second sound, the excitation energy from the condensate $\epsilon_{p}=\omega_{p}-\omega_{0}$ has the same velocity as $c_{2}\left(1-\left(T / T_{\lambda}\right)\right)^{1 / 3}+D\left(1-\left(T / T_{\lambda}\right)\right)^{1 / 2}$. For a large momentum $p, \epsilon_{p}$ is proportional to $p^{2}$ because the kinetic energy occupies a main part of the total energy. Consequently, the function form is

$$
\epsilon_{p}=p c_{2}\left(1-\left(T / T_{\lambda}\right)+a p^{3}\right)^{1 / 3}+p D\left(1-\left(T / T_{\lambda}\right)+b p^{2}\right)^{1 / 2},
$$

where $c_{2}$ and $D$ are determined from data of the second sound velocity, and $a$ and $b$ are parameters. This form is used in the phonon region $\left(0<p<\hbar q_{1}\right)$.

We can calculate the specific heat $C_{\mathrm{P}}$ as follows.

$$
\begin{aligned}
C_{\mathrm{P}}=T\left(\frac{\partial S}{\partial T}\right)_{\mathrm{P}}= & T k_{B} \frac{1}{(2 \pi \hbar)^{3}}\left(\frac{\partial V}{\partial T}\right)_{\mathrm{P}} \int_{0}^{\infty}\left[\log \left(1+n_{p}\right)+\frac{\epsilon_{p}}{k_{B} T} n_{p}\right] 4 \pi p^{2} \mathrm{~d} p \\
& +T k_{B} \frac{V}{(2 \pi \hbar)^{3}} \int_{0}^{\infty} \frac{\epsilon_{p}}{k_{B} T}\left(\frac{\partial n_{p}}{\partial T}\right)_{\mathrm{P}} 4 \pi p^{2} \mathrm{~d} p .
\end{aligned}
$$

In this calculation, a logarithmic singularity appears in the last integration $Y$ as

$$
\begin{aligned}
Y & =\int_{0}^{\infty} \frac{\epsilon_{p}}{k_{B} T}\left(\frac{\partial n_{p}}{\partial T}\right)_{\mathrm{P}} 4 \pi p^{2} \mathrm{~d} p \\
& =\int_{0}^{\infty} \frac{\epsilon_{p}}{k_{B} T}\left(\frac{-\exp \left(\frac{\epsilon_{p}}{k_{B} T}\right)}{\left(\exp \left(\frac{\epsilon_{p}}{k_{B} T}\right)-1\right)^{2}}\right)\left(\frac{1}{k_{B} T}\right)\left(\frac{\partial \epsilon_{p}}{\partial T}\right)_{\mathrm{P}} 4 \pi p^{2} \mathrm{~d} p+\cdots
\end{aligned}
$$

Therein we can use the following approximations:

$$
\begin{aligned}
& \left(\frac{\partial \epsilon_{p}}{\partial T}\right)_{\mathrm{P}} \approx p c_{2} \frac{1}{3} \frac{(-1)}{T_{\lambda}}\left(1-\left(T / T_{\lambda}\right)+a p^{3}\right)^{-2 / 3} \text { and } \\
& \left(\exp \left(\frac{\epsilon_{p}}{k_{B} T}\right)-1\right)^{-2} \approx\left(\frac{\epsilon_{p}}{k_{B} T}\right)^{-2}
\end{aligned}
$$


Fig. 2 (Color on-line) Specific heat $C_{\mathrm{P}}$ of liquid helium II near the $\lambda$ point $[\mathrm{J} /($ mole $\mathrm{K})]$

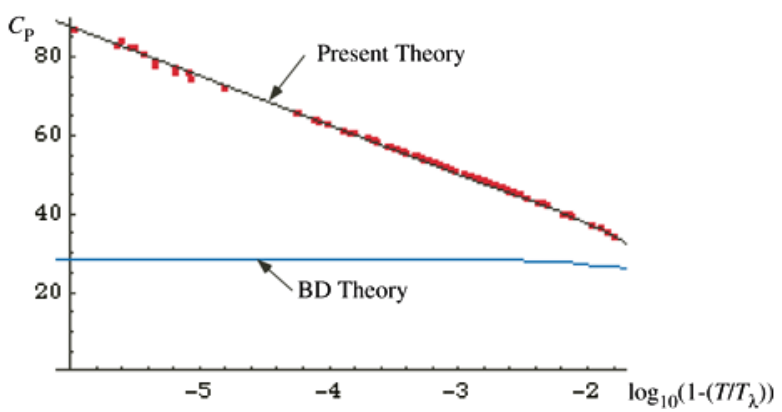

for a small $p$ near $T_{\lambda}$. Then, we obtain

$$
\begin{aligned}
Y & \approx \int_{0}^{p_{1}} \frac{1}{3 T_{\lambda}} \frac{1}{\left(1-\left(T / T_{\lambda}\right)+a p^{3}\right)} 4 \pi p^{2} \mathrm{~d} p+(\text { regular term }) \\
& =\int_{0}^{a p_{1}^{3}} \frac{4 \pi}{9 a T_{\lambda}} \frac{1}{\left(1-\left(T / T_{\lambda}\right)+a p^{3}\right)} \mathrm{d}\left(a p^{3}\right)+(\text { regular term }) .
\end{aligned}
$$

This integration has a logarithmic singularity as $Y=-\frac{4 \pi}{9 a T_{\lambda}} \log \left(1-\left(T / T_{\lambda}\right)\right)+$ (regular term).

On the other hand, the singularity disappears in BD theory because of non zero value of the phonon velocity at the $\lambda$ point. We evaluate the integrations in $C_{\mathrm{P}}$ for the temperature range $0<\left(1-\left(T / T_{\lambda}\right)\right)<0.02$, the results of which are shown in Fig. 2. The upper curve expresses our calculated values of specific heat. Dots in Fig. 2 express the experimental data $[3,4]$ of specific heat for a saturated vapor pressure. Our calculation is performed under the value of pressure $P=0.05$ bar. The difference between saturated vapor pressure and $P=0.05$ bar is negligibly small. The lower curve in Fig. 2 expresses results of the BD theory. As the figure illustrates, our result has logarithmic divergence at the $\lambda$ point. Moreover, the present results agree well with the experimental data. On the other hand, the BD theory curve depicts no divergent behavior. This result means that the second sound mode in Brillouin scattering near the $\lambda$ point is the elementary excitation of the dressed boson from the Bose-Einstein condensate.

\section{References}

1. J. Bendt, R.D. Cowan, J.L. Yarnell, Phys. Rev. 113, 1386 (1959)

2. J.S. Brooks, R.J. Donnelly, J. Phys. Chem. Ref. Data 6, 51 (1977)

3. G. Ahlers, Phys. Rev. A 3, 696-716 (1971)

4. M.J. Buckingham, W.F. Fairbank, in Progress in Low Temperature Physics, vol. 3, ed. by C.J. Gorter (Amsterdam, North-Holland, 1961), p. 80

5. G. Winterling, F.S. Holmes, T.J. Greytak, Phys. Rev. Lett. 30, 427 (1973)

6. G. Winterling, J. Miller, T.J. Greytak, Phys. Lett. 48A, 343 (1974)

7. W.F. Vinen, C.J. Palin, J.M. Vaughan, in Proc. of 13th Conf. on Low Temperature Physics (1972), p. 524

8. J.M. Vaughan, W.F. Vinen, C.J. Palin, in Proc. of 13th Conf. on Low Temperature Physics (1972), p. 532

9. S. Sasaki, in Springer Series in Solid State Science, vol. 79 (1989), p. 160

10. D.S. Greywall, G. Ahlers, Phys. Rev. A 7, 2145 (1973)

글 Springer 